\title{
Molecular identification, characterization, and expression analysis of a novel trypsin inhibitor-like cysteine-rich peptide from the cotton bollworm, Helicoverpa armigera (Hübner) (Lepidoptera: Noctuidae)
}

\author{
Muhammad Shakeel* (iD and Junaid Zafar
}

\begin{abstract}
Trypsin inhibitor-like cysteine-rich domain (TIL)-type protease inhibitors have been reported to inhibit proteases such as trypsin, cathepsin, elastase, and chymotrypsin, and thus play a critical role in several physiological processes. However, the information about TIL peptides in insects is limited. In the present study, a novel cysteine-rich trypsin inhibitor-like protease, designated as HaTIL2, was isolated from the cotton bollworm, Helicoverpa armigera (Hübner) (Lepidoptera: Noctuidae). The CDNA sequence of HaTIL2 is 470 nucleotides long, with 240 nucleotides open reading frame encoding 80 amino acid residues. The analysis of genomic DNA revealed that the full-length genomic DNA sequence of HaTIL2 was 574 bp with two exons and one intron. The predicted molecular weight of HaTIL2 is $8.632 \mathrm{kDa}$, with an isoelectric point of 4.41. The results of neighbor-joining tree demonstrated that HaTIL2 was closely related to H. armigera TIL3, DmCEl, and DsCtAPI followed by TCIMI and MdCEl. The mRNA of HaTIL2 was constitutively expressed at different levels in different stages of $\mathrm{H}$. armigera. The HaTIL2 showed a high expression on different days of the pupal stage, which revealed that HaTIL2 might play a vital role during the pupal stage. Although the detailed function of HaTIL2 needs to be elucidated, the obtained results are of particular importance to open up new avenues of research into the functional studies of insect peptidase inhibitors.
\end{abstract}

Keywords: Trypsin inhibitor-like domain, Cysteine-rich peptide, Helicoverpa armigera, mRNA expression, Serine protease inhibitors

\section{Background}

Protease inhibitors are classified either by the type of proteases they inhibit, such as serpins, cystatins, and metalloproteases, or according to the presence of structural motifs such as Kunitz, Kasal, or Bowman-Birk (Song et al. 2008). Peptides containing trypsin inhibitorlike domain (TIL) are usually comprised of 56-84 amino residues and known to play a key role in the various physiological processes by inhibiting proteinases (Nie et al. 2012). Generally, TIL-type protease contains a single TIL domain with 10 cysteine residues, which form 5

\footnotetext{
* Correspondence: faizaneabiwaqas@scau.edu.cn

Key Laboratory of Bio-Pesticide Innovation and Application of Guangdong

Province, College of Agriculture, South China Agricultural University, Guangzhou, China
}

disulfide bridges (Bania et al. 1999), and shows inhibitory activities against trypsin, cathepsin, chymotrypsin, or elastase. A few TIL domain peptides reported until now include BmKAPi from Mesobuthus martensii Karsch (Zeng et al. 2002), BMSI7 from Boophilus microlpus (Fogaca et al. 2006), SjAPI from Scorpiops jendeki (Chen et al. 2013), APim6 from Apis melifera (Michel et al. 2012), AcCI from Apis cerana (Kim et al. 2013), and BtCI from Bombus terrestris (Qiu et al. 2012). The TIL domain identified from the venom of a scorpion species, Scorpiops jendeki, shows dual function peptide with $\alpha$ chymotrypsin- and elastase-inhibiting properties (Chen et al. 2013). Similarly, BMSI isolated from hemocytes of B. microplus was reported to inhibit chymotrypsin and elastase (Fogaca et al. 2006). In a frog species, 
Lepidobatrachus laevis, a TIL domain cysteine-rich peptide was isolated from the skin and showed inhibitory activity against trypsin (Wang et al. 2015). In A. cerana, it was identified that the recombinant AcCI demonstrated inhibitory activity against chymotrypsin (Kim et al. 2013). It has been reported that in Bombyx mori, mutations at the second and sixth cysteine residue dramatically reduced the activity of inhibition against microbial proteases (Li et al. 2016). Though a couple of studies are available on TIL domain-containing cysteinerich peptides from insects, however, until now, to the best of our knowledge, there is no information available for such kind of peptides in $H$. armigera.

$H$. armigera is one of the most destructive polyphagous cosmopolitan insect pest species (Noor-ul-Ane et al. 2018). Over recent decades, several pests have developed resistance against various families of synthetic insecticides (Shakeel et al. 2017). Similarly, H. armigera has also evolved resistance to several insecticides, and resistance might be a factor accountable for its pest status (Wu 2007). This has led to developing alternative control tactics (Sun 2015). Therefore, it is imperative to identify and understand the molecular mechanism of such genes that might play a critical role during various physiological functions of insects.

Considering the importance of TIL domain cysteine-rich peptides in physiological processes, as exhibited by previous reports, the present study aimed to identify and describe the bioinformatic analysis of a cysteine-rich peptide of $H$. armigera including cloning, isolation of genomic DNA, and investigation of the expression pattern in the fat body of $H$. armigera, especially at the pupal stage. Furthermore, the phylogenetic relationship of $H$. armigera TIL domain cysteine-rich peptides to TIL domain peptides from other insects, was studied.

\section{Materials and methods}

\section{Insect culture}

$H$. armigera population was nurtured on an artificial diet (Abbasi et al. 2007) under the laboratory conditions of $25 \pm 2{ }^{\circ} \mathrm{C}, 70 \% \mathrm{RH}$, and a photoperiod of 16:8-h L:D.
Identification of HaTIL2 cDNA and nucleotide sequencing The sequence-specific primers were designed in accordance with the length of the cDNA sequence of HaTIL2 to amplify the open reading frame (ORF) (Table 1). Then, automated DNA sequencer (Perkin-Elmer Applied Biosystems, Foster City, CA, USA) was used to sequence the plasmid DNA. The sequence was then compared, using DNASIS and BLAST databanks of NCBI. The amino acid sequence was aligned by MacVector (ver. 6.5, Oxford Molecular Ltd.).

\section{Biochemical properties and phylogenetic tree construction}

NCBI Basic Protein BLAST was used to identify homologous TIL domain sequences from different insects with $H$. armigera HaTIL2 sequence used as the query. PEPSTATS was used to compute the primary sequence composition of IPK2 (Rice et al. 2000). The physicochemical parameters of HaTIL2 were predicted using the ProtParam tool (Gasteiger et al. 2005). The SignalP 4.1 server was used to identify the possible secretory signal peptide of HaTIL2 (Petersen et al. 2011). To detect the evolutionary location and phylogenetic similarities of HaTIL2 with other insect TIL domain peptides, multiple sequence alignment (MSA) of selected amino acid sequences was carried out to produce quality alignments. To better understand the evolutionary relationship of HaTIL2 with other TIL domain peptides, serpin sequences of $H$. armigera, Tribolium castaneum, Musca domestica, Drosophila miranda, Drosophila suzukii, A. florea, Bombus ignitus, B. terrestris, B. impatiens, and A. cerana were used to construct a phylogenetic tree, using the maximum parsimony method in MEGA (Tamura et al. 2013). Tree \#1 out of 2 most parsimonious trees (length $=223$ ) is shown. The consistency index was (0.870968), the retention index was (0.800000), and the composite index was $0.699552(0.696774)$ for all sites and parsimony-informative sites (in parentheses). The percentage of replicate trees in which the associated taxa clustered together in the bootstrap test (1000 replicates) is shown next to the branches. The MP tree was obtained using the Subtree-Pruning-Regrafting (SPR)

Table 1 Sequences of PCR and qPCR primers used in study of Helicoverpa armigera

\begin{tabular}{ll}
\hline Gene name & Sequence \\
\hline HaTIL2-F & 5'-GGATCCATGTTTAAGTCGTCTTTATTGATTTTGAG-3' \\
HaTIL2-R & 5'GGTACCTTAATGATGATGATGATGATGCTGGGGGCAGTCATCAATAGCTATGCATTCTCC-3' \\
HaTIL2-qF & 5'-GTAGTGCTTGTCCAACTA-3' \\
HaTIL2-qR & 5'-CTCCATCGTCATTCTTG-3' \\
RPL28-qF & 5'-CATCTGAACTGGATGATC-3' \\
RPL28-qR & 5'-GTACACTACTGTGAAACC-3' \\
\hline
\end{tabular}


algorithm with search level 1 in which the initial trees were obtained by the random addition of sequences (10 replicates). The analysis involved 11 amino acid sequences. All positions containing gaps and missing data were eliminated. There were 70 positions in the final dataset. Finally, iTOL was used to render the final image (Letunic and Bork 2016).

\section{Genomic DNA isolation and PCR}

The FGENESH program (Salamov and Solovyev 2000) was used to predict the number of potential exons and introns in the HaTIL2 genomic DNA. For experimental verification, the whole bodies of $H$. armigera $(n=3)$ were used to isolate DNA (Wizard Genomic DNA Purification Kit, Promega ${ }^{\circ}$. The cDNA sequence of HaTIL2 was used to design the amplification primers. The verification of PCR products was done by DNA sequence analysis.

\section{Cloning of genes from $\mathrm{H}$. armigera}

To amplify gene-specific primers, cDNA and a set of sense and antisense primers were used in PCR (Table 1). For the digestion reaction, sense primer was incorporated into the BamHI restriction site, whereas to incorporate antisense primer, the KpnI restriction site was used. The amplification by PCR had the following steps: denaturation at $95{ }^{\circ} \mathrm{C}$ for $3 \mathrm{~min}$, then 35 cycles at $95{ }^{\circ} \mathrm{C}$ for $30 \mathrm{~s}, 55^{\circ} \mathrm{C}$ for $30 \mathrm{~s}$, and $72{ }^{\circ} \mathrm{C}$ for $2 \mathrm{~min}$, with a final extension at $72{ }^{\circ} \mathrm{C}$ for $5 \mathrm{~min}$. The PCR product was visually examined on a $1 \%(\mathrm{w} / \mathrm{v})$ agarose gel stained with ethidium bromide using the Bio-Rad imaging system. The gel extraction kit (Omega) was used to purify the target gene-amplified product. The purified product was then ligated to pGEM-T easy vector (Takara) and transformed into Escherichia coli bacteria DH5 $\alpha$. A positive clone was then selected on LB agar plates that contained $50 \mu \mathrm{g} \mathrm{mL}{ }^{-1}$ ampicillin after incubation at $37{ }^{\circ} \mathrm{C}$ overnight. The resulting PCR clones were sequenced by Shanghai Sunny Biotech Co., Ltd.

\section{Collection of tissues}

Dissection of healthy $H$. armigera larvae was performed on the ice under a stereomicroscope (Zeiss, Jena, Germany). The insect fat body was collected and washed with PBS (140 mM NaCl, $27 \mathrm{mM} \mathrm{KCl,} 8 \mathrm{mM} \mathrm{Na} 2 \mathrm{HPO}$, and $1.5 \mathrm{mM} \mathrm{KH} 2 \mathrm{PO} 4, \mathrm{pH}$ 7.4). The fat body tissues were stored at $-80{ }^{\circ} \mathrm{C}$ for RNA extraction.

\section{Total RNA preparation and synthesis of CDNA template for real-time qPCR}

The isolation of the entire RNA was done using TRIzol reagent following the manufacturer's protocol. The assessment of RNA sample quality was performed by running on $1 \%(\mathrm{w} / \mathrm{v})$ agarose gel electrophoresis. The UV spectrophotometer was used to assess the concentration of RNA samples. DNase I (Fermentas) eliminated the contamination of DNA. The synthesis of cDNA was carried out using reverse transcription RevertAid ${ }^{\mathrm{TM}}$ Reverse Transcriptase (Fermentas) in a $20-\mu \mathrm{L}$ reaction. Finally, the resultant product was stored at $-80{ }^{\circ} \mathrm{C}$.

\section{Fluorescence real-time quantitative PCR analysis of gene expression}

To perform real-time qPCR, a Bio-Rad iQ2 cycler was used and the reaction was carried out in PCR strips. The amplification signal was detected by using SYBR Green chemistry. Triplicate first-strand DNA aliquots for each sample served as templates for RT-qPCR using the SsoFast $^{\text {tw }}$ EvaGreen $^{\circ}$ Supermix (Bio-Rad, Hercules, CA, USA) with an iQ2 Optical System (Bio-Rad). Each reaction was carried out in $\mathrm{iQ}^{\mathrm{m}}$ 96-well PCR plates, $100 \mathrm{nM}$ of each primer and a $20-\mu \mathrm{L}$ volume of cDNA, covered with adhesive seals (Bio-Rad). The thermal reaction was as follows: denaturation for $30 \mathrm{~s}$ at $95{ }^{\circ} \mathrm{C}$, then 40 cycles for $5 \mathrm{~s}$ at $95{ }^{\circ} \mathrm{C}$, and $60{ }^{\circ} \mathrm{C}$ for $10 \mathrm{~s}$. Furthermore, a melting curve analysis was carried out for the amplified product. To analyze the relative expression, the $2^{-\Delta \Delta C T}$ method (Livak and Schmittgen 2001) was employed with a ribosomal protein L28 (RPL28) used as an internal control for normalization (Chandra et al. 2014; Shakeel et al. 2015, 2018). The list of primers for RT-qPCR is presented in Table 1.

\section{Results and discussion}

The TIL domain found in protease inhibitors has been documented to inhibit chymotrypsin, elastase, cathepsin, and trypsin, and thus play an important role in various biological processes. Several protease inhibitors with a TIL domain have been identified previously (Zeng et al. 2002; Michel et al. 2012; Qiu et al. 2012; Chen et al. 2013; Kim et al. 2013). However, currently, there were no other publications about the identification and expression analysis of $H$. armigera TIL domain cysteinerich peptides; thus, this work represents the first report about identification, cloning, and expression analysis of a novel TIL domain cysteine-rich peptide, designated as HaTIL2.

\section{Sequence analysis of HaTIL2 and CDNA cloning}

In the present study, based on the sequence-specific primers of HaTIL2, 470-bp fragment was amplified, which contained ORF of 240 nucleotides encoding 80 amino acid residues. The deduced amino acid sequence analysis showed that HaTIL2 had predicted the molecular weight of $8.632 \mathrm{kDa}$ with an isoelectric point of 4.41 . Obtained results of molecular weight of HaTIL2 were similar to other documented TIL domain peptides (Zeng 
et al. 2002; Michel et al. 2012; Qiu et al. 2012; Chen et al. 2013 and Kim et al. 2013).

\section{Analysis of HaTIL2 genomic DNA sequence}

It has been documented that introns play a vital role in gene expression and the introduction of an intron into a gene elevated the level of gene expression (Le Hir et al. 2003; Nott et al. 2003). In the present study, genomic DNA sequence analysis was conducted to determine the number of introns and exons present in the HaTIL2 gene of $H$. armigera. For this purpose, the FGENESH software was used to predict the HaTIL2 gene sequence. The results of sequence prediction revealed that the fulllength genomic DNA sequence of HaTIL2 has two exons and one intron. To confirm the presence of two exons and one intron in the HaTIL2 sequence, genomic DNA was isolated from $H$. armigera and used as a template for PCR. The amplification results showed an amplicon size of 574 nucleotides. The PCR products were verified by DNA sequence analysis, which also demonstrated one intron and two exons in HaTIL2. The gain of an intron by TIL domain proteases might be during the evolution process (Zeng et al. 2014). As TIL peptides are also involved in the immune response of insects, gain of intron might give rise to stronger immune response. The TIL family is categorized as a group of inhibitors having low molecular weight and a TIL domain consisting of a high proportion of cysteine residues (Rosengren et al. 2001). In a previous study, it was documented that trypsin sequences contained 7 cysteine residues (Bown et al. 1997), which were lower than those observed in this study, as 11 cysteine residues in HaTIL2 domain peptide were found. The presence of cysteine residues in odd numbers might indicate difference in the activity of inhibition (Li et al. 2016).

\section{Biochemical properties of HaTIL2}

The biochemical properties of HaTIL2 predicted by ProtParam revealed Cys $(13.8 \%)$ as the most abundant amino acid, followed by Val (8.8\%), Ser (8.8\%), Asp (7.5\%), and Ala (6.2\%); Trp (0.0\%) and Met (1.2\%) were the least abundant amino acids in the HaTIL2 sequence (Table 2). The isoelectric point (pI) of HaTIL2 was approximately 5 , indicating that it might be soluble in the acidic buffer. Instability indices (Ii) are used to determine the in vivo half-lives, and a protein with an Ii value lower than 40 is predicted to be stable (Rogers et al. 1986). The Ii of HaTIL2 was computed to be 58.89 indicating that it might be thermally unstable. Moreover, the present study obtained a high aliphatic index (80.38) for the HaTIL2 protein. The gradient average hydropathicity (GRAVY) analysis was conducted to determine the hydrophobicity of HaTIL2, whereby a positive GRAVY value indicates a hydrophobic nature and negative
Table 2 Biochemical properties of the HaTIL2 protein sequence of Helicoverpa armigera

\begin{tabular}{ll}
\hline Properties & Values \\
\hline Number of amino acids & 80 \\
Molecular weight & 8632.80 \\
Theoretical pl & 4.41 \\
Amino acid composition (\%) & \\
Ala & 6.2 \\
Arg & 3.8 \\
Asn & 5.0 \\
Asp & 7.5 \\
Cys & 13.8 \\
Gln & 5.0 \\
Glu & 5.0 \\
Gly & 6.2 \\
His & 1.2 \\
Ile & 6.2 \\
Leu & 6.2 \\
Lys & 2.5 \\
Met & 1.2 \\
Phe & 1.2 \\
Pro & 2.5 \\
Ser & 80.89 \\
Thr & 0.089 \\
Trp & \\
Tyr & 3.8 \\
Valiphatic index & 5.0 \\
\hline
\end{tabular}

GRAVY value, a hydrophilic nature (Kyte and Doolittle 1982). The GRAVY index for HaTIL2 was calculated to be 0.089 (Table 2) indicating that HaTIL2 is hydrophobic and cannot interact favorably with water. The SignalP 4.1 server identified the presence of a signal peptide at the $\mathrm{N}$-terminus location of the protein indicating that HaTIL2 is a secretory protein that might play a critical role in the inhibition of extracellular SPs. Previously, several SPIs have been reported to contain the potential signal peptide and mature peptide (Bania et al. 1999; Cierpicki et al. 2000; Kim et al. 2013).

\section{Multiple sequence alignment of HaTIL2}

When HaTIL2 was compared with the other members, the multiple sequence alignment showed that HaTIL2 had $83.33 \%$ identity with HaTIL3 ( $H$. 


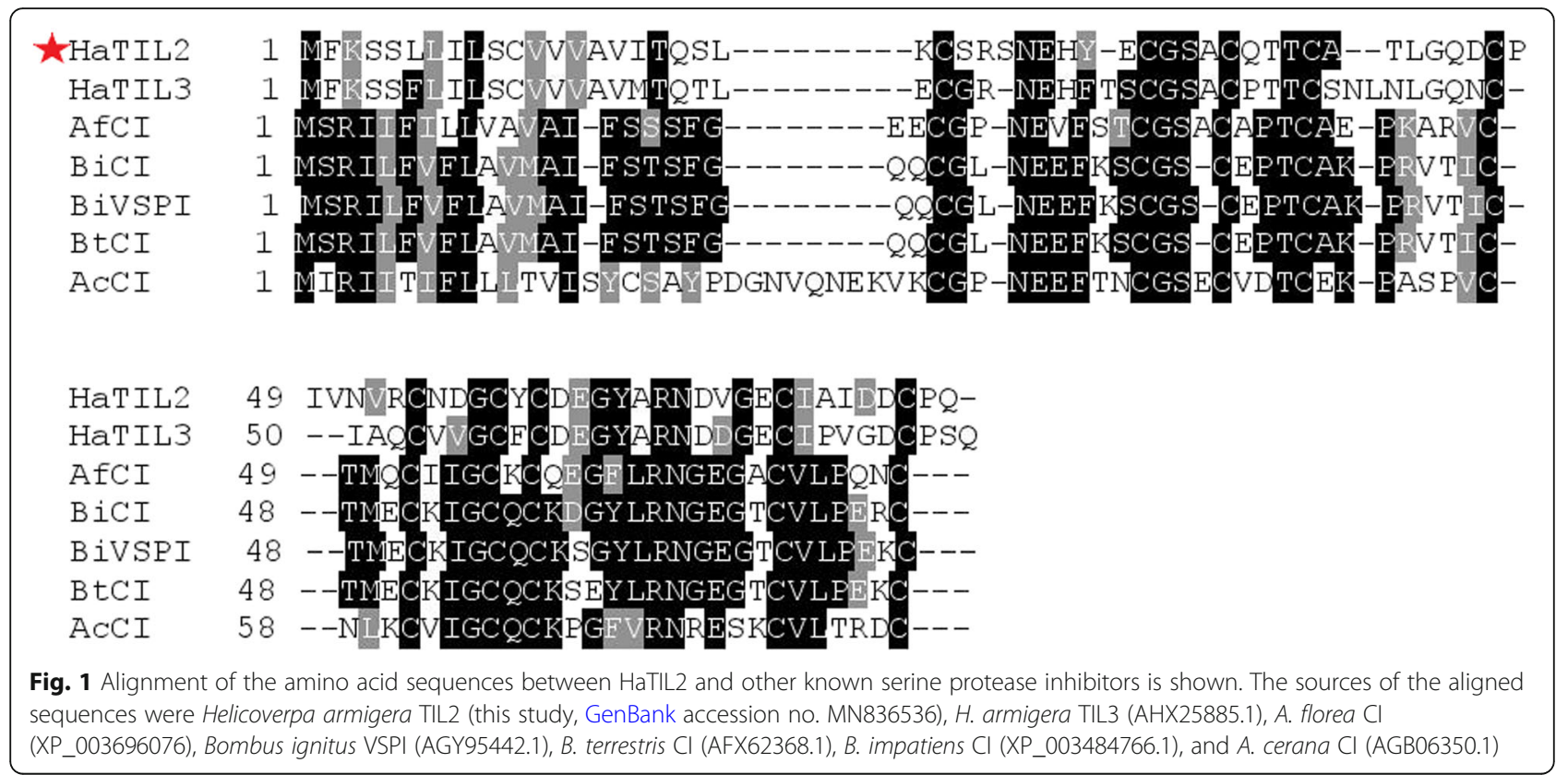

armigera) and 36\% identity with AfCI (Apis florea), which was greater than that of $34 \%$ identity with $\mathrm{BiCI}$ and BiVSPI (Bombus ignitus), 32\% identity with BtCI (Bombus terrestris), and 31\% identity with AcCI (Apis cerana) (Fig. 1). The results of HaTIL2 multiple sequence alignment indicated a low identity level (3136\%) with other TIL domain peptides, and such phenomenon was not surprising as this sort of heterogeneity was common among serpins. It has been demonstrated that six serpins of Manduca sexta exhibited a very low level of amino acid identity (40\%) within the species (Tong and Kanost 2005).
Phylogenetic tree analysis of HaTIL2

To get insight into the evolutionary relationships of HaTIL2 with cysteine-rich peptides from other insects, a neighborjoining tree was constructed using maximum parsimony analysis. The results of neighbor-joining tree demonstrated that HaTIL2 is in the same clade as HaTIL3 (H. armigera), DmCEI (D. miranda), DsCtAPI (D. suzukii), TcIMI (T. castaneum), and $\operatorname{MdCEI~(M.~domestica)~(Fig.~2).~}$

The mRNA expression profile of HaTIL2 in the fat body In order to characterize the biological role of HaTIL2 in $H$. armigera, the expression pattern of mRNA was

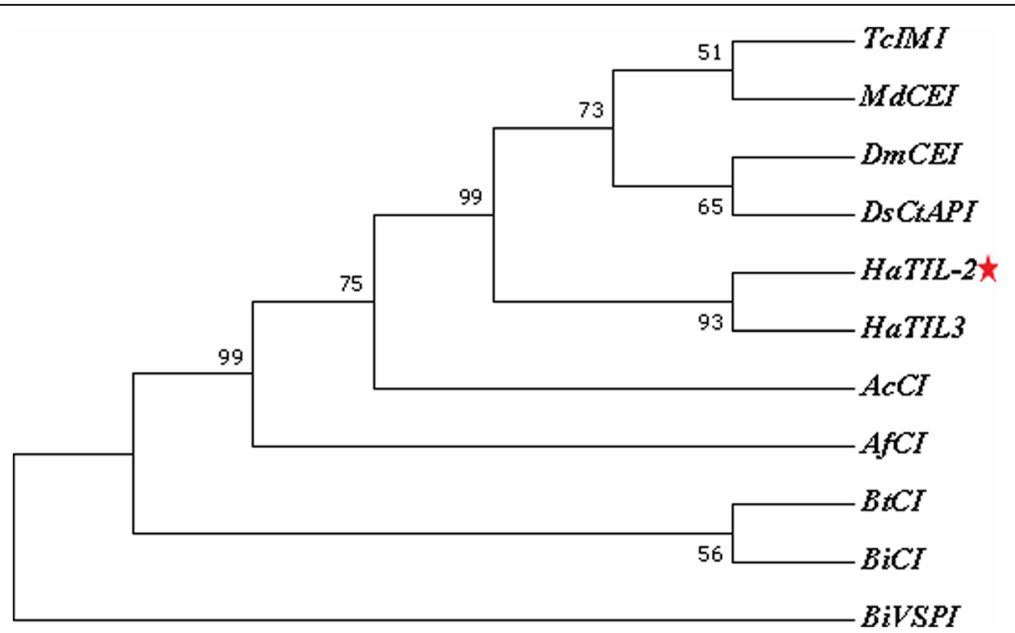

Fig. 2 Phylogenetic relationship of HaTIL2 with cysteine-rich proteases of other insects. The sources of the sequences were Helicoverpa armigera TIL2 (this study, GenBank accession no. MN836536), H. armigera TIL3 (AHX25885.1), Tribolium castaneum IMI (XP_008201444.1), Musca domestica CEI (XP_005177283.1), Drosophila miranda CEI (XP_017134624.1), Drosophila suzukii CtAPI (XP_016937015.1) A. florea Cl (XP_003696076), Bombus ignitus VSPI (AGY95442.1), B. terrestris Cl (AFX62368.1), B. impatiens CI (XP_003484766.1), and A. cerana AcCl (AGB06350.1) 


\section{Expression profile of HaTIL2 in fat body}

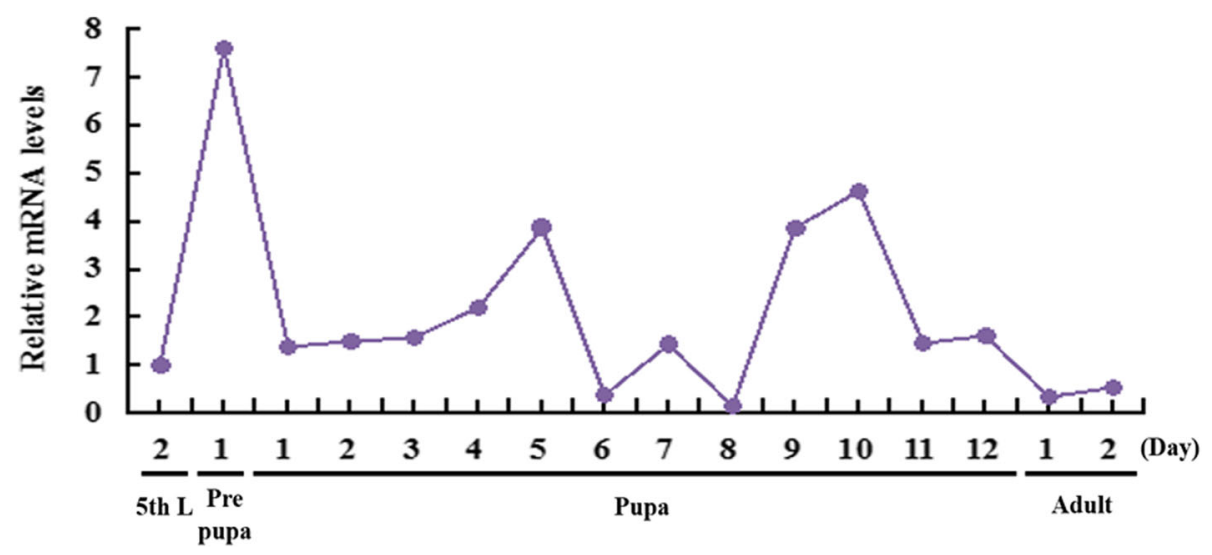

Fig. 3 Expression profiles of HaTIL2 in Helicoverpa armigera fat body tissue are shown. The expression profile of HaTIL2 in the fat body during H. armigera development was analyzed by RT-qPCR. The ribosomal protein L28 gene was used as the internal control for RT-qPCR

investigated in the fat body at different stages of $H$. armigera especially at the pupal stage. For this purpose, the SYBER Green real-time PCR was employed on cDNA of $H$. armigera fat body tissue to determine tissue-specific mRNA expression of HaTIL2. The RPL28 gene was used as an endogenous control. The mRNA transcripts of HaTIL2 were expressed constitutively in the fat body at larval, prepupal, pupal, and adult stages (Fig. 3). The consistent expression of HaTIL2 was observed in the fat body indicating that HaTIL2 is a serine protease inhibitor derived from the $H$. armigera body. As HaTIL2 was consistently expressed in the fat body, it shows that the fat body is the major site of serine protease inhibitor genes. Our results are also in accordance with the studies of Chamankhah et al. (2003), Li et al. (2012) and Liu et al (2015), where serine protease inhibitor genes were highly expressed in the fat body. The HaTIL2 showed high expression at the prepupal stage and 5th, 8th, 9th, and 10th days of pupal stage, whereas it was low on other days of the pupal stage and at the adult stage (Fig. 3) suggesting that it might also play an important role during the pupal stage.

\section{Conclusion}

This is the first study to identify a cysteine-rich serine protease inhibitor from the fat body of $H$. armigera. Obtained results are of particular interest, as a new avenue of research towards functional studies of protease inhibitors will be opened. Further studies should be conducted to find out the detailed functional role of protease inhibitors in $H$. armigera.

\section{Abbreviations}

BLAST: Basic local alignment search tool; bp: Base pair; cDNA: Complementary deoxyribonucleic acid; Cl: Chymotrypsin inhibitor; DNA: Deoxyribonucleic acid; kDa: Kilodaltons; LB: Luria Bertani;
mRNA: Messenger ribonucleic acid; NCBI: National Center for Biotechnology Information; ORF: Open reading frame; PCR: Polymerase chain reaction; QPCR: Quantitative polymerase chain reaction; RH: Relative humidity; RTqPCR: Real-time quantitative polymerase chain reaction; TIL: Trypsin inhibitorlike

\section{Acknowledgements}

We are thankful to the anonymous reviewers for their useful comments on the initial draft of the manuscript.

\section{Authors' contributions}

MS designed the study, MS supervised the work, MS and JZ wrote the manuscript, and MS carried out the experiments. MS and JZ revised the initial draft. The authors read and approved the final manuscript.

\section{Funding}

This work was supported by a grant from Postdoctoral Science Foundation of China (2018M640788).

\section{Availability of data and materials}

All the data has been included in the manuscript.

Ethics approval and consent to participate

Not applicable

\section{Consent for publication}

Not applicable

\section{Competing interests}

The authors declare that they have no competing interests.

Received: 12 September 2019 Accepted: 19 January 2020

Published online: 03 February 2020

\section{References}

Abbasi BH, Ahmed K, Khalique F, Ayub N, Liu HJ, Kazmi SAR, Aftab MN (2007) Rearing the cotton bollworm, Helicoverpa armigera, on a tapioca-based artificial diet. J Insect Sci 7:35

Bania J, Stachowiak D, Polanowski A (1999) Primary structure and properties of the cathepsin $\mathrm{G} /$ chymotrypsin inhibitor from the larval hemolymph of Apis mellifera. Eur J Biochem 262:680-687

Bown DP, Wilkinson HS, Gatehouse JA (1997) Differentially regulated inhibitorsensitive and insensitive protease genes from the phytophagous insect pest, Helicoverpa armigera, are members of complex multigene families. Insect Biochem Mol Biol 27:625-638 
Chamankhah M, Braun L, Visal-Shah S, O'Grady M, Baldwin D, Shi X, Hemmingsen S, Alting-Mees M, Hegedus D (2003) Mamestra configurata serpin-1 homologues: cloning, localization and developmental regulation. Insect Biochem Mol Biol 33:355-369

Chandra GS, Asokan R, Manamohan M, Kumar NK, Sita T (2014) Evaluation of reference genes for quantitative real-time PCR normalization in cotton bollworm Helicoverpa armigera. Mol Biol 48:813-822

Chen Z, Wang B, Hu J, Yang W, Cao Z, Zhuo R, Li W, Wu Y (2013) SjAPI, the first functionally characterized Ascaris-type protease inhibitor from animal venoms. PLoS One 8:e57529

Cierpicki T, Bania J, Otlewski J (2000) NMR solution structure of Apis mellifera chymotrypsin/cathepsin G inhibitor-1 (AMCl-1): structural similarity with Ascaris protease inhibitors. Protein Sci 9:976-984

Fogaca AC, Almeida IC, Eberlin MN, Tanaka AS, Bulet P, Daffre S (2006) Ixodidin, a novel antimicrobial peptide from the hemocytes of the cattle tick Boophilus microplus with inhibitory activity against serine proteinases. Peptides 27:667-674

Gasteiger E, Hoogland C, Gattiker A, Wilkins MR, Appel RD, Bairoch A (2005) Protein identification and analysis tools on the ExPASy server. In: Walker J.M. (eds) The Proteomics Protocols Handbook. Humana Press

Kim BY, Lee KS, Wan H, Zou FM, Choi YS, Yoon HJ, Kwon HW, Je YH, Jin BR (2013) Anti-elastolytic activity of a honeybee (Apis cerana) chymotrypsin inhibitor. Biochem Biophys Res Commun 430:144-149

Kyte J, Doolittle RF (1982) A simple method for displaying the hydropathic character of a protein. J Mol Biol 157:105-132

Le Hir H, Nott A, Moore MJ (2003) How introns influence and enhance eukaryotic gene expression. Trends Biochem Sci 28:215-220

Letunic I, Bork P (2016) Interactive tree of life (iTOL) v3: an online tool for the display and annotation of phylogenetic and other trees. Nucleic Acids Res $44: 242-245$

Li Y, Liu H, Zhu R, Xia Q, Zhao P (2016) Loss of second and sixth conserved cysteine residues from trypsin inhibitor-like cysteine-rich domain-type protease inhibitors in Bombyx mori may induce activity against microbial proteases. Peptides 86:13-23

Li Y, Zhao P, Liu S, Dong Z, Chen J, Xiang Z, Xia Q (2012) A novel protease inhibitor in Bombyx mori is involved in defense against Beauveria bassiana. Insect Biochem Mol Biol 42:766-775

Liu D, Wang L, Yang L, Qian C, Wei G, Dai L, Li J, Zhu B, Liu C (2015) Serpin-15 from Bombyx mori inhibits prophenoloxidase activation and expression of antimicrobial peptides. Dev Comp Immunol 51:22-28

Livak KJ, Schmittgen TD (2001) Analysis of relative gene expression data using real-time quantitative $P C R$ and the $2-\Delta \Delta C T$ method. Methods 25:402-408

Michel Y, Mclntyre M, Ginglinger H, Ollert M, Cifuentes L, Blank S, Spillner E (2012) The putative serine protease inhibitor Api m 6 from Apis mellifera venom: recombinant and structural evaluation. J Investig Allergol Clin Immunol 22: 476-484

Nie Y, Zeng XC, Luo X, Wu S, Zhang L, Cao H, Zhou J, Zhou L (2012) Tremendous intron length differences of the BmKBT and a novel BmKBT-like peptide genes provide a mechanical basis for the rapid or constitutive expression of the peptides. Peptides 37:150-156

Noor-ul-Ane M, Mirhosseini MA, Crickmore N, Saeed S, Noor I, Zalucki MP (2018) Temperature-dependent development of Helicoverpa armigera (Hübner)(Lepidoptera: Noctuidae) and its larval parasitoid, Habrobracon hebetor (Say)(Hymenoptera: Braconidae): implications for species interactions. Bull Entomol Res 108:295-304

Nott A, Meislin SH, Moore MJ (2003) A quantitative analysis of intron effects on mammalian gene expression. RNA 9:607-617

Petersen BT, Chennat J, Cohen J, Cotton PB, Greenwald DA, Kowalski TE, Krinsky ML, Park WG, Pike IM, Romagnuolo J (2011) Multisociety guideline on reprocessing flexible gastrointestinal endoscopes. Gastrointest Endosc 73: 1075-1084

Qiu Y, Yoon HJ, Jin BR (2012) Molecular cloning and characterization of chymotrypsin inhibitor and chitin-binding protein homologs from the bumblebee Bombus terrestris. Int J Ind Entomol 25:115-121

Rice P, Longden I, Bleasby A (2000) EMBOSS: the European molecular biology open software suite. Trends Genet 16:276-277

Rogers S, Wells R, Rechsteiner M (1986) Amino acid sequences common to rapidly degraded proteins: the PEST hypothesis. Science 234:364-368

Rosengren KJ, Daly NL, Scanlon MJ, Craik DJ (2001) Solution structure of BSTI: a new trypsin inhibitor from skin secretions of Bombina bombina. Biochemistry 40:4601-4609
Salamov AA, Solovyev W (2000) Ab initio gene finding in Drosophila genomic DNA. Genome Res 10:516-522

Shakeel M, Farooq M, Nasim W, Akram W, Khan FZA, Jaleel W, Zhu X, Yin H, Li S, Fahad S (2017) Environment polluting conventional chemical control compared to an environmentally friendly IPM approach for control of diamondback moth, Plutella xylostella (L), in China: a review. Environ Sci Pollut Res 24:14537-14550

Shakeel M, Rodriguez A, Tahir UB, Jin F (2018) Gene expression studies of reference genes for quantitative real-time PCR: an overview in insects. Biotechnol Lett 40:227-236

Shakeel M, Zhu X, Kang T, Wan H, Li J (2015) Selection and evaluation of reference genes for quantitative gene expression studies in cotton bollworm, Helicoverpa armigera (Lepidoptera: Noctuidae). J Asia Pac Entomol 18:123-130

Song G, Zhou M, Chen W, Chen T, Walker B, Shaw C (2008) HV-BBI - a novel amphibian skin Bowman-Birk-like trypsin inhibitor. Biochem Biophys Res Commun 372:191-196

Sun X (2015) History and current status of development and use of viral insecticides in China. Viruses 7:306-319

Tamura K, Stecher G, Peterson D, Filipski A, Kumar S (2013) MEGA6: molecular evolutionary genetics analysis version 60. Mol Biol Evol 30:2725-2729

Tong Y, Kanost MR (2005) Manduca sexta serpin-4 and serpin-5 inhibit the prophenol oxidase activation pathway cDNA cloning, protein expression, and characterization. J Biol Chem 280:14923-14931

Wang YW, Tan JM, Du CW, Luan N, Yan XW, Lai R, Lu QM (2015) A novel trypsin inhibitor-like cysteine-rich peptide from the frog Lepidobatrachus laevis containing proteinase-inhibiting activity. Nat Prod Bioprospect 5:209-214

Wu K (2007) Monitoring and management strategy for Helicoverpa armigera resistance to Bt cotton in China. J Invertebr Pathol 95:220-223

Zeng X-C, Liu Y, Shi W, Zhang L, Luo X, Nie Y, Yang Y (2014) Genome-wide search and comparative genomic analysis of the trypsin inhibitor-like cysteine-rich domain-containing peptides. Peptides 53:106-114

Zeng XC, Wang SX, Li WX (2002) Identification of BmKAPi, a novel type of scorpion venom peptide with peculiar disulfide bridge pattern from Buthus martensii Karsch. Toxicon 40:1719-1722

\section{Publisher's Note}

Springer Nature remains neutral with regard to jurisdictional claims in published maps and institutional affiliations.

\section{Submit your manuscript to a SpringerOpen ${ }^{\circ}$ journal and benefit from:}

- Convenient online submission

Rigorous peer review

- Open access: articles freely available online

- High visibility within the field

- Retaining the copyright to your article

Submit your next manuscript at $\boldsymbol{\nabla}$ springeropen.com 